\title{
Erratum to: Respiratory viruses in patients and employees in an intensive care unit
}

\author{
D. Stupica $\cdot$ L. Lusa $\cdot$ M. Petrovec $\cdot$ \\ N. Žigon • M. Jevšnik · P. Bogovič • \\ F. Strle
}

Published online: 16 March 2012

(C) Springer-Verlag 2012

\section{Erratum to: Infection \\ DOI 10.1007/s15010-012-0245-6}

Unfortunately some errors occurred in the published version.

Page 2, line 8: "all" should be omitted:

Study participants were evaluated at 16 different timepoints during the study period, namely, on each Monday and Thursday for 8 consecutive weeks between 12 January and 5 March 2009.

Page 2, line 23: "all" should be omitted:

Nasopharyngeal swabs and plasma specimens were collected from participants at ach of the 16 predetermined study time-points for the detection of selected respiratory viruses.

Page 3, line 14: "considered to be" should be omitted:

Nine patients were not included in the study because their stay in the ICU was too short.

The online version of the original article can be found under doi:10.1007/s15010-012-0245-6.

D. Stupica $(\bowtie) \cdot$ P. Bogovič · F. Strle

Department of Infectious Diseases, University Medical Center Ljubljana, Japljeva 2, 1525 Ljubljana, Slovenia

e-mail: cerar.dasa@gmail.com

L. Lusa

Medical Faculty, Institute for Biostatistics and Medical

Informatics, University of Ljubljana, Ljubljana, Slovenia

M. Petrovec · N. Žigon · M. Jevšnik

Medical Faculty, Institute of Microbiology and Immunology,

University of Ljubljana, Ljubjana, Slovenia
Page 3, line 27: "most" should be replaced with "more"

Page 3, line 28-29: "with a concomitant infection by" should be omitted:

The detection of INF A and $\mathrm{CoV}$ in the nasopharyngeal swab was associated more frequently with a concomitant lower respiratory tract infection than the other viruses (Table 2).

Page 4, Fig. 1: It would be more representative if Fig. 1 was drawn across the full width of the paper sheet. Fig. 1a and Fig. 1b should be exactly aligned one above the other because the calendar dates are the same in Fig. 1a and Fig. $1 b$.

Page 5, right column, line 2: one parenthesis should be omitted:

Although the majority of our patients were hospitalized in the ICU due to an infectious cause, the frequency of viruspositive respiratory specimens among our patient cohort (which varied from 0 to $36.4 \%$ over the study period, median 10.6\%, IQR 9.1-27.1\%; Fig. 1b) was lower than that found in these cited studies.

Page 5, right column, line 8: "the only" should be omitted: In our study, the detection of respiratory viruses in nasopharyngeal swabs was not always associated with a lower respiratory tract infection, as demonstrated by 11 of 31 (35.48\%, 95\% CI 19.83-54.62\%) virus-positive patients without lower respiratory tract infection.

Acknowledgments We thank Maja Sočan, MD, PhD (Communicable Disease Centre, National Institute of Public Health, Ljubljana, Slovenia) for kindly providing data on the incidence of influenza-like illness in the community of Ljubljana region. 